\title{
Corrigendum: Passively mode-locked laser with an ultra-narrow spectral width
}

Michael Kues, Christian Reimer, Benjamin Wetzel, Piotr Roztocki, Brent E. Little, Sai T. Chu, Tobias Hansson, Evgeny A. Viktorov, David J. Moss and Roberto Morandotti

Nature Photonics 11, 159-162 (2017); published online 23 January 2017; corrected after print 1 August 2017.

In the version of this Letter originally published, in the Acknowledgements, the following information was unavailable at the time of publication: "B.E.L. was supported by the Strategic Priority Research Program of the Chinese Academy of Sciences, grant no. XDB24030000." This information has now been added in the online versions of the Letter.

\section{Corrigendum: Single-photon imager based on a superconducting nanowire delay line}

Qing-Yuan Zhao, Di Zhu, Niccolò Calandri, Andrew E. Dane, Adam N. McCaughan, Francesco Bellei, Hao-Zhu Wang, Daniel F. Santavicca and Karl K. Berggren

Nature Photonics 11, 247-251 (2017); published online 27 March 2017; corrected after print 14 August 2017.

In the version of this Article originally published, in Fig. $3 c$, in the $y$ axis label, the units '(c.p.s.)' should not have been included; the label should have read "Dark counts". This has now been corrected in the online versions of the Article. 